\title{
Comparative analysis of the influence of Fructus Ligustri Lucidi on a rat lumbar disc herniation model
}

\author{
YA-XIN HAN, DONG LIANG, XIAO-RUI HAN and DE-YONG LIANG
}

Department of Orthopedics, The First Affiliated Hospital of China Medical University, Shenyang, Liaoning 110001, P.R. China

Received May 26, 2014; Accepted March 2, 2015

DOI: $10.3892 / \mathrm{mmr} .2015 .3547$

\begin{abstract}
Lumbar disc herniation (LDH) is a term used for a group of conditions, including back pain, femoral nerve pain and sciatica. Currently available treatments and surgical options are insufficient for patients with $\mathrm{LDH}$. Fructus Ligustri Lucidi (FLL) is a herb that is used for treating age-associated diseases. The results of the present study suggested that FLL may be used for treatment of patients with LDH. In the present study, matrix metalloproteinase-1, -3, -8 and -9 (MMP-1, -3, -8 and -9) protein and mRNA expression downregulation was observed in patients with LDH according to western blotting and reverse transcription-quantitative polymerase chain reaction. By contrast, upregulation of interleukin-2 (IL-2), IL-6, IL-8 and tumor necrosis factor- $\alpha$ (TNF- $\alpha$ ) expression was observed in patients with $\mathrm{LDH}$, according to an enzyme-linked immunosorbent assay. Mechanical allodynia was observed in rats with LDH not treated with FLL; however, not in FLL-treated rats. IL-2, IL-6, IL-8 and TNF- $\alpha$ expression levels in the serum from untreated rats were significantly higher than that of the FLL-treated rat models. Protein expression levels of MMPs in FLL-treated rats were lower than those in untreated rats. However, the mechanisms underlying the association between FLL and protein expression levels require further investigation.
\end{abstract}

\section{Introduction}

Fructus Ligustri Lucidi (FLL), the vernacular name for the fruit of Ligustrum lucidum, is a herb used for treating age-associated diseases (1). FLL ethanol extract modulates the turnover of bone and the calcium balance in ovariectomized rats (2). FLL may improve the uptake of calcium

Correspondence to: Dr Ya-Xin Han, Department of Orthopedics, The First Affiliated Hospital of China Medical University, 155 Nanjing North Street, Heping, Shenyang, Liaoning 110001, P.R. China E-mail: doctor_yaxin@163.com

Key words: lumbar disc herniation, fructus ligustri lucidi, matrix metalloproteinases, inflammatory cytokines, gene ontology from the diet, predominantly via its actions on increasing the levels of 1,25-dihydroxyvitamin $\mathrm{D}_{3}\left(1,25(\mathrm{OH})_{2} \mathrm{D}_{3}\right)$ serum and vitamin $\mathrm{D}$-dependent calcium binding proteins (CaBP) (3).

Lower back pain is one of the most prevalent and costly health problems in developed countries. For example, lower back pain was found to be one of the most expensive diseases in Australia, with an estimated cost in 2001 of $\$ 9.17$ billion (4). The predominant cause of lower back pain is lumbar disc herniation ( $\mathrm{LDH}) . \mathrm{LDH}$ is a term referring to a group of conditions, including back pain, femoral nerve pain and sciatica. LDH may be caused by the compression of dural or spinal nerve roots, associated with rupturing of the annulus fibrosus or by a herniated nucleus forcing pressure on the spinal canal (5). Current treatments and surgeries for patients with LDH include conventional open discectomy, microdiscectomy, percutaneous laser discectomy, percutaneous discectomy and microendoscopic discectomy (6). Although there are numerous cases with successful outcomes for patients with LDH following disc surgery, there remain a significant number of patients who do not benefit from this procedure $(7,8)$.

To the best of our knowledge, little is known as to whether FLL may improve the prognosis for patients with LDH following disc surgery. In the present study, the efficacy of FLL to reduce pain was evaluated using a lumbar disc herniation rat model.

\section{Materials and methods}

Tissue samples. Intervertebral disc tissue samples were collected from 51 patients who had undergone posterior open discectomy for LDH in the Department of Orthopedics (The First Affiliated Hospital of China Medical University, Shenyang, China) between May 2010 and May 2013. Five cadaveric tissue samples were also obtained from cancer patients within $36 \mathrm{~h}$ of mortality with no previously known spinal pathology. Blood samples from the patients with LDH and 20 healthy volunteers were collected in ethylenediamine tetraacetic acid on ice. Samples were immediately centrifuged at 3,000 x g for $20 \mathrm{~min}$. Plasma samples were frozen at $-70^{\circ} \mathrm{C}$. Patients or relatives signed an informed consent form approved by the China Medical University ethics committee prior to participation in the study. The study design was in accordance with the Declaration of Helsinki. Patient and control group data are provided in Table I. 
Cytokine assay. Enzyme-linked immunosorbent assay (ELISA) kits for inflammatory cytokines: Interleukin-2 (IL-2), IL-6, IL-8 and tumor necrosis factor- $\alpha$ (TNF- $\alpha$ ) were purchased from R\&D Systems, Inc. (Minneapolis, MN, USA). ELISA was conducted according to the manufacturer's instructions. Cytokine levels were expressed as $\mathrm{pg} / \mathrm{ml}$ of sample homogenate.

Preparation of FLL extract. Ligustrum lucidum plants were obtained from Liaoning, China. Dried and powered crude plant material was extracted twice using $70 \%$ ethanol. The crude plant was boiled with distilled water twice, each lasting $2 \mathrm{~h}$. The mixture was filtered to collect the filtrate, which was evaporated using a rotary evaporator (rotary evaporator; Shyarong Biochemical Instruments Inc., Shanghai, China) under reduced pressure, yielding $23.5 \%$ of the weight of the starting materials.

Operation procedure. The study protocol was approved by the Animal Ethics Committee of China Medical University. Male Sprague-Dawley rats (n=90; weight, 200-250 g; Harlan Sprague Dawley Inc.; Indianapolis, IN, USA) were used in the present study. According to methods described previously by Obata $e t a l$, rats were anesthetized using intraperitoneal injection of sodium pentobarbital $(40 \mathrm{mg} / \mathrm{kg}$; Beijing Propbs Biotechnology Co., Ltd., Beijing, China) (9). Laminectomies were performed in the left L5 nerve roots and dorsal root ganglions (DRG) were exposed. Nucleus pulposuses, harvested from the second and third coccygeal intervertebral discs, were implanted next to the left L5 nerve roots, near to the DRG.

Treatment groups. Rats were separated into three groups: Control group, normal diet; mock group, normal diet mixed with ethanol $(200 \mathrm{mg} / \mathrm{kg})$ and FLL group, normal diet mixed with FLL extract $(200 \mathrm{mg} / \mathrm{kg})$. Rats were provided with sterile deionized water, ad libitum.

Evaluation of mechanical allodynia and thermal hyperalgesia. Evaluations were performed prior to surgery (day 0) and on days 5, 10, 15, 20, 25 and 30 after surgery.

Mechanical allodynia was evaluated by measuring the withdrawal response of the hind paw to a mechanical stimulation, using a Von Frey assay (North Coast Medical, Inc., Gilroy, CA, USA) that had been calibrated to the force required to elicit a withdrawal response (g) (10). Rats were placed in a clear plastic cage with a metal mesh floor and allowed to acclimatize to the testing environment for $15 \mathrm{~min}$. The plantar surface of each hind paw was stimulated five times using Von Frey filaments, beginning with a $0.1 \mathrm{~g}$ filament, the thickness was increased or decreased, until a withdrawal response was observed in three of the five stimuli. Filaments were increased every 6-8 mins with logarithmically incremental rigidity of $0.41,0.70,1.20$, $2.00,3.63,5.50,8.50$ and $15.1 \mathrm{~g}$, to calculate the mechanical threshold. Fifty percent probability thresholds of mechanical paw withdrawal were calculated. If no withdrawal response of the hind paw was observed prior to stimulation with a $26-\mathrm{g}$ filament, $26 \mathrm{~g}$ was assigned as the mechanical threshold.

Thermal hyperalgesia was determined by measuring paw withdrawal latency in a thermal stimulation system (XR1102; Shanghai Xin Ruan Information Technology Co.,
Table I. Patient characteristics.

\begin{tabular}{lcc}
\hline Feature & $\begin{array}{c}\text { Control } \\
\text { group }\end{array}$ & $\begin{array}{c}\text { LDH } \\
\text { group }\end{array}$ \\
\hline $\begin{array}{l}\text { Male/Female (n) } \\
\text { Mean age (years) }\end{array}$ & $4 / 1$ & $38 / 13$ \\
$\begin{array}{l}\text { Body mass index } \\
\text { Mean }\end{array}$ & 40 & 42 \\
Range & 27.7 & 26.8 \\
Position of herniated disc & $21.5-33.2$ & $20.22-31.25$ \\
L3-L4 & & \\
L4-L5 & NA & 15 \\
L5-S1 & NA & 18 \\
Type of herniation & NA & 18 \\
Protrusion & & \\
Extrusion & NA & 22 \\
Sequestration & NA & 18 \\
Duration of symptoms & NA & 11 \\
$<3$ months & & \\
$3-12$ months & NA & 7 \\
$>12$ months & NA & 19 \\
Pain intensity (visual analog scale) & & 25 \\
$0-5$ & NA & \\
$5-7$ & NA & 15 \\
$8-10$ & NA & 16 \\
\hline
\end{tabular}

LDH, lumber disc herniation.

Ltd., Shanghai, China) consisting of a clear plastic chamber $(10 \times 20 \times 24 \mathrm{~cm})$ on a clear smooth glass floor, at $30^{\circ} \mathrm{C}$. Rats were placed individually in the chamber for $\sim 15 \mathrm{~min}$, in order to acclimatize to the chamber conditions. A heat stimulus $\left(150 \mathrm{mcal} / \mathrm{sec} / \mathrm{cm}^{2}\right)$ was delivered using a $0.5-\mathrm{cm}$ diameter radiant heat source positioned under the plantar surface of the paw. Once a rat withdrew its paw from the heat stimulus, a photocell detected the interruption of a light beam, which automatically switched off the infrared generator and stopped the timer, providing the value for paw withdrawal latency. This method exhibits a $0.1 \mathrm{sec}$ precision level for the measurement of paw withdrawal latency. If a rat failed to withdraw its paw the heat stimulus was automatically discontinued after $25 \mathrm{sec}$.

\section{Reverse transcription-quantitative polymerase chain reaction} $(R T-q P C R)$. Total tissue RNA was isolated using TRIzol ${ }^{\circledR}$ reagent (Invitrogen Life Technologies, Carlsbad, CA, USA) and was reverse transcribed using SuperScript II reverse transcriptase (Invitrogen Life Technologies) according to the manufacturer's instructions. RT-qPCR analysis was performed using an ABI prism 7500 sequence detection system (Applied Biosystems Life Technologies, Foster, CA, USA) and an SYBR Green PCR Master mixture (Takara Biotechnology Co., Ltd., Dalian, China). Primer sequences are shown in Table II. PCR conditions were as follows: One cycle at $95^{\circ} \mathrm{C}$ for $10 \mathrm{~min}$ followed by 40 cycles of $95^{\circ} \mathrm{C}$ for $15 \mathrm{sec}$ and $60^{\circ} \mathrm{C}$ for $1 \mathrm{~min}$. 
Relative quantification was calculated by the $\Delta \Delta \mathrm{Ct}$ method using High Resolution Melt v.2.0 software from Applied Biosystems Life Technologies.

Western blotting. Specimens from the patients and the mouse model were lysed using a lysis buffer $(50 \mathrm{mM}$ Tris- $\mathrm{HCl}$, pH 7.4; 150 mM NaCl, $1 \%$ Triton X-100, $0.1 \%$ sodium dodecyl sulfate, $1 \mathrm{mM}$ ethylenediaminetetraacetic acid, $1 \mathrm{mM} \mathrm{Na} \mathrm{VO}_{4}$ and $1 \mathrm{mM} \mathrm{NaF}$, protease inhibitor cocktail). The extracts were incubated on ice for $20 \mathrm{~min}$ and centrifuged at $12,000 \mathrm{x} \mathrm{g}$ for $20 \mathrm{~min}$ at $4^{\circ} \mathrm{C}$, and the supernatants were collected. Protein concentrations were determined using a Bradford assay (Bio-Rad Laboratories, Hercules, CA, USA) and proteins were separated using 10\% Bis-Tris gel (Bio-Rad Laboratories) electrophoresis, transferred to a nitrocellulose membrane (Bio-Rad Laboratories), and western blot analysis was performed. The primary antibodies are summarized in Table III. The secondary mouse monoclonal antibodies antibodies included anti-mouse IgG (A0216), anti-rabbit IgG (A0239) or anti-goat IgG (A0182; determined by primary antibodies) at a dilution of 1:1,000-2,000 (Amersham Biosciences, Needham, MA, USA). Subsequently, the results were detected by enhanced chemiluminescence (Amersham Pharmacia, Piscataway, NJ, USA).

GeneChip technology. Total RNA was extracted from cells as described above. Total RNA samples were then analyzed using a GeneChip assay (Affymetrix, Inc., Santa Clara, CA, USA). Three replicates were performed for each experimental group. Gene expression analysis was performed using GeneChip (Affymetrix), according to the manufacturer's instructions. Gene expression analysis was performed using three arrays and three independent mRNA samples for each treatment. Microarray data were analyzed using Bio MAS 3.0 software (CapitalBio Corporation, Beijing, China). A fold change of $\geq 2$ or $\leq 0.5$ and a Q-value of $\leq 5 \%$ were used as cutoff criteria The value of the control group was set as a standard of 1.0 and the value of the other two groups was plotted with respect to the control group. Differentially expressed genes were screened and clustered among the control group, mock group and FLL group using an Affymetrix Rat230.2 array.

Gene ontology $(G O)$ analysis. The associations between gene expression and biological processes, molecular functions, and cellular compartments, were annotated using the GOTree Machine (11). GOTree Machine uses a hypergeometric test to evaluate the significance of gene enrichment for each category by determining whether the observed number of gene counts exceed the expected counts. Results were visualized as a directed acyclic graph in order to demonstrate the relationships among the enriched GO categories.

Statistical analysis. Using GraphPad 5.0 software (GraphPad Software, Inc., La Jolla, CA, USA), data were analyzed using Student's t-test. $\mathrm{P}<0.05$ was considered to indicate a statistically significant difference.

\section{Results}

MMP and inflammatory cytokine levels in healthy and $\mathrm{LDH}$ tissues. MMP-1, -3, -8, -9 and -13 protein expression levels
Table II. Primers used in RT-qPCR analyses.

\begin{tabular}{lc}
\hline Gene & Sequence $\left(5^{\prime}-3^{\prime}\right.$, forward and reverse) \\
\hline MMP-1 & GGCCCACAAACCCCAAAAG \\
MMP-3 & ATCTCTGTCGGCAAATTCGTAAGC \\
& GATGCCCACTTTGATGATGATGAA \\
MMP-8 & AGTGTGGCTGAGTGAAAGAGACC \\
& ATCAAATGTCAAACTGGGGTCAC \\
MMP-9 & TGCCCGGACCAAGGATACAGTTT \\
MMP-13 & AGGCCGTGGCTCAGGTTCAGG \\
& CCCCAACCCTAAACATCCAAAAAC \\
GAPDH & TTAAAAACAGCTCCGCATCAACCT \\
& ATGCCAGTGAGCTTCCCGTTCAGC \\
\hline
\end{tabular}

RT-qPCR, reverse transcription-quantitative polymerase chain reaction; MMP, metalloproteinase.

Table III. Antibodies used in western blot analysis.

\begin{tabular}{lcc}
\hline Protein & Catalogue number & Dilution \\
\hline MMP-1 & ab52631 & $1: 100$ \\
MMP-3 & ab18898 & $1: 100$ \\
MMP-8 & ab81286 & $1: 100$ \\
MMP-9 & ab38898 & $1: 100$ \\
MMP-1 & ab39012 & $1: 100$ \\
$\beta$-actin & ab8227 & $1: 500$ \\
\hline
\end{tabular}

MMP, metalloproteinase.

were downregulated in healthy tissues compared with those in LDH tissues ( $\mathrm{P}<0.05$, Fig. 1A). Transcript levels of MMPs were significantly higher in LDH tissues than in healthy tissues $(\mathrm{P}<0.05$, Fig. 1B). Inflammatory cytokine expression levels were significantly lower in the healthy control group compared with those in the LDH group (Fig. $1 \mathrm{C} ; \mathrm{P}<0.05$ ).

Effects of FLL on mechanical allodynia and thermal hyperalgesia. Paralysis was not observed in rats throughout the experiments. Significant attenuation of mechanical allodynia was observed in the FLL-treated group compared with the control and mock groups $(\mathrm{P}<0.05$, Fig. $2 \mathrm{~A})$. Mean withdrawal latency in rats in the FLL-treated group was significantly higher than in rats in the control and mock groups $(\mathrm{P}<0.05$, Fig. 2B). IL-2, IL-6, IL- 8 and TNF- $\alpha$ expression levels were significantly higher in the control and mock group compared with those in the FLL-treated group ( $\mathrm{P}<0.05$; Fig. $2 \mathrm{C}$ ). Protein expression levels of MMPs in the FLL-treated group were lower than those in the mock and control groups (Fig. 2D).

Affymetrix GeneChip analysis. The expression of 645 rat transcripts was analyzed using the GeneChip Rat Genome 
A
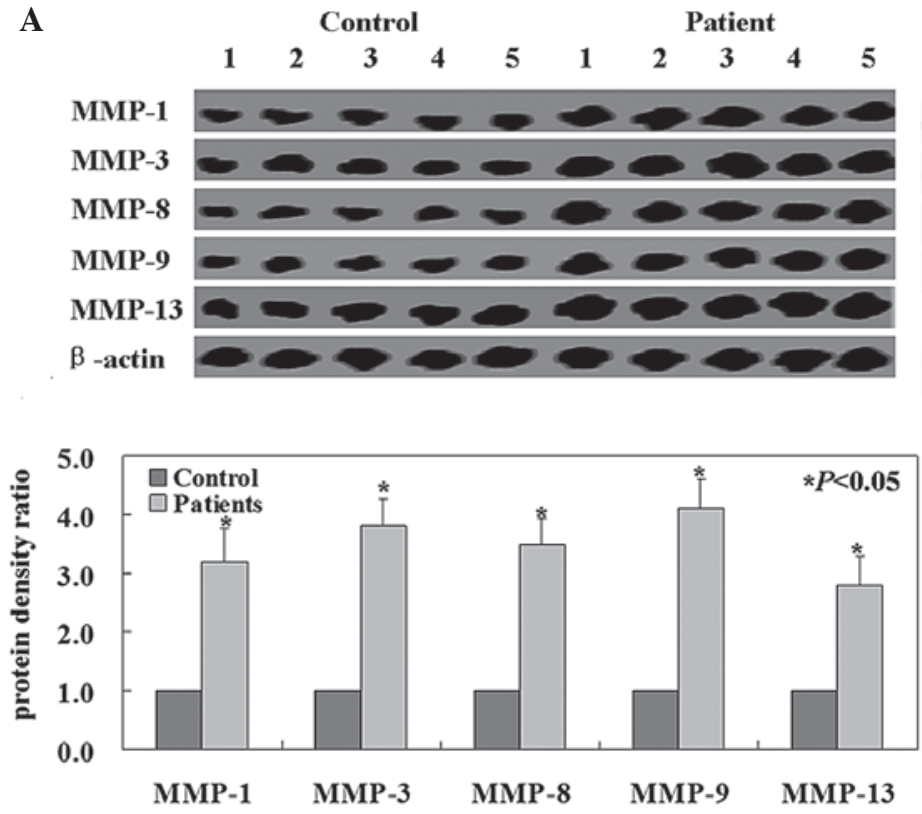

B

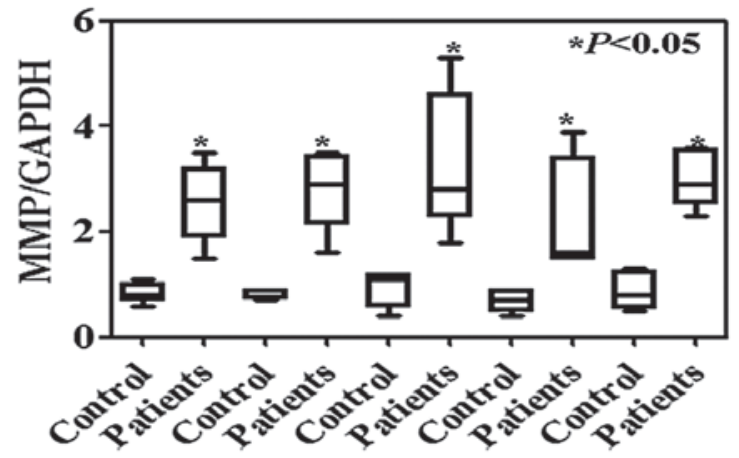

C MMP-1 MMP-3 MMP-8 MMP-9 MMP-13

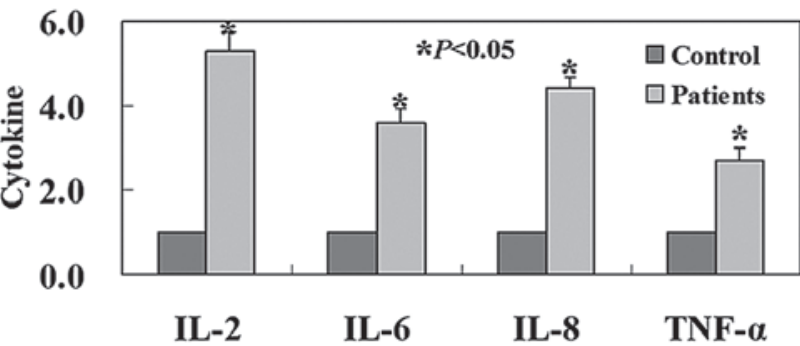

Figure 1. MMP expression in control and patient tissue samples. (A) Representative results of MMP protein expression in LDH tissues and healthy tissues according to western blot analysis. $\beta$-actin was used as an internal control. (B) MMP mRNA expression was measured in specimens by using reverse transcription quantitative-polymerase chain reaction. GADPH was used as an internal control. (C) Results of an enzyme-linked immunosorbent assay demonstrated the levels of inflammatory cytokines in serum from the healthy control group and the patients with LDH. LDH, lumbar disc herniation; MMP, metalloproteinase.

A

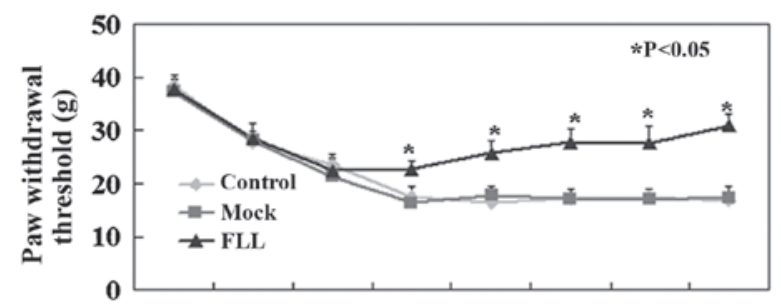

C

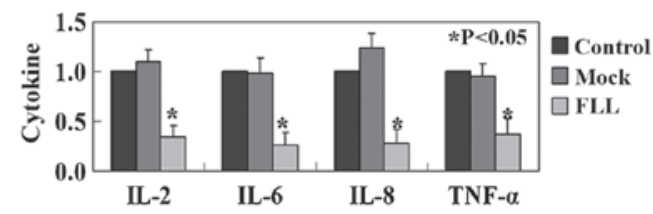

B

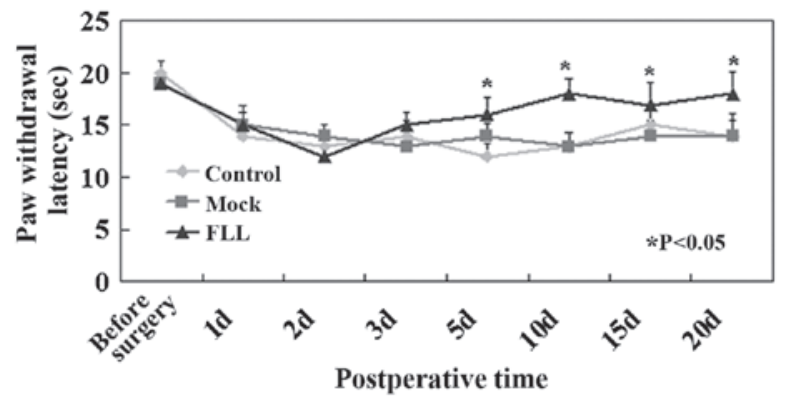

D

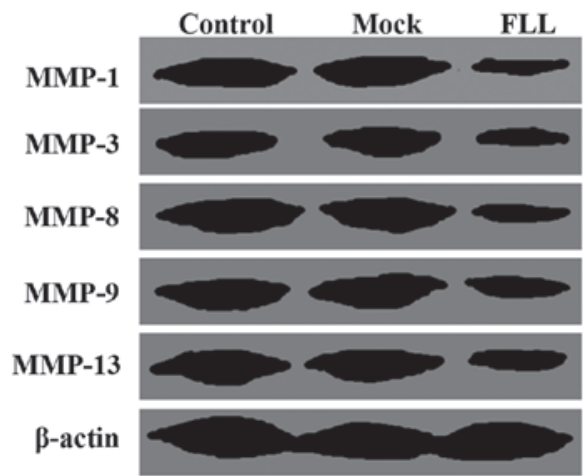

Figure 2. Rats in the mock and control groups exhibited (A) significant mechanical allodynia and (B) lower paw withdrawal latency compared with the FLL group. (C) The levels of inflammatory cytokines were measured using enzyme-linked immunosorbent assay. (D) Expression of MMPs was measured using western blot analysis. MMP, metalloproteinase; IL, interleukin.

array (Affymetrix). The expression of 125 genes was upregulated and that of 345 genes was downregulated in the FLL-treated group compared with the control and mock groups ( $\mathrm{P}<0.05$, Fig. 3). A GO analysis was conducted using the GOTree Machine. The GO analysis produced clusters of enriched differentially expressed genes $(\mathrm{P}<0.01)$ in three 

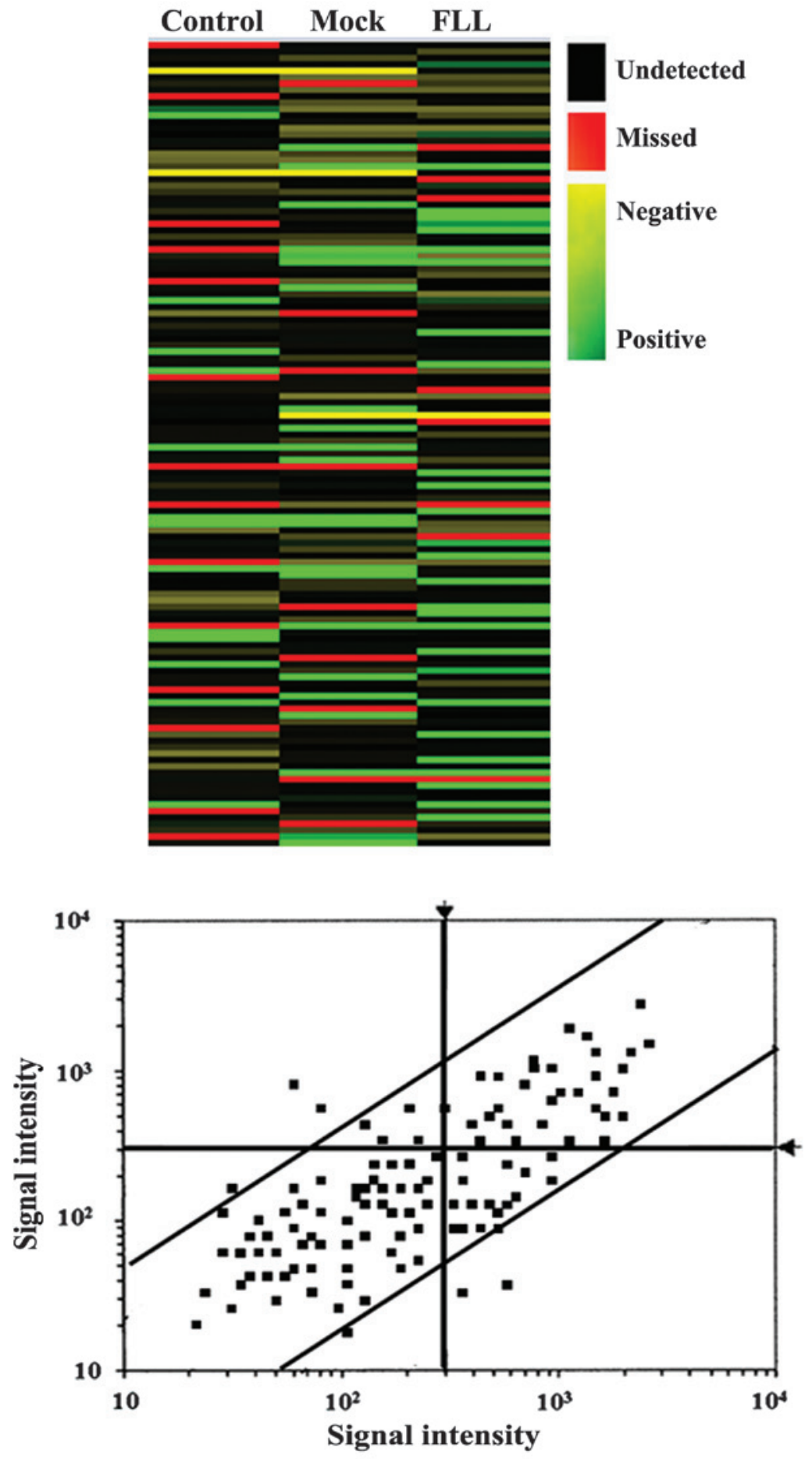

Figure 3. Microarray analysis identified a number of subtle differences in RNA expression. Distribution of duplicate hybridizations showing average normalized ratio signal intensities for microarray. Green indicates higher values and yellow indicates lower values.

categories: Biological processes, cellular component and molecular function. In the GO terms tree (Figs. 4 and 5) the association and overlap of MMPs and inflammatory cytokines are demonstrated. In total, enriched GO terms were found in 71 subcategories under biological process, 16 subcategories under cellular component and 51 subcategories under molecular function. In biological process ontology, the results indicated that cytokine production, cellar localization, protein secretion are predominantly associated with FLL treatment.

\section{Discussion}

In the present study, downregulation of the protein expression of MMPs and upregulation of IL-2, IL-6, IL- 8 and TNF- $\alpha$ expression was observed in the patients with $\mathrm{LDH}$ compared with that in control group. Weiler et al (12) demonstrated a significant correlation between MMP protein activity and histological signs of degeneration in patients with LDH. Similarly, Tsarouhas et al (13) found higher MMP mRNA 

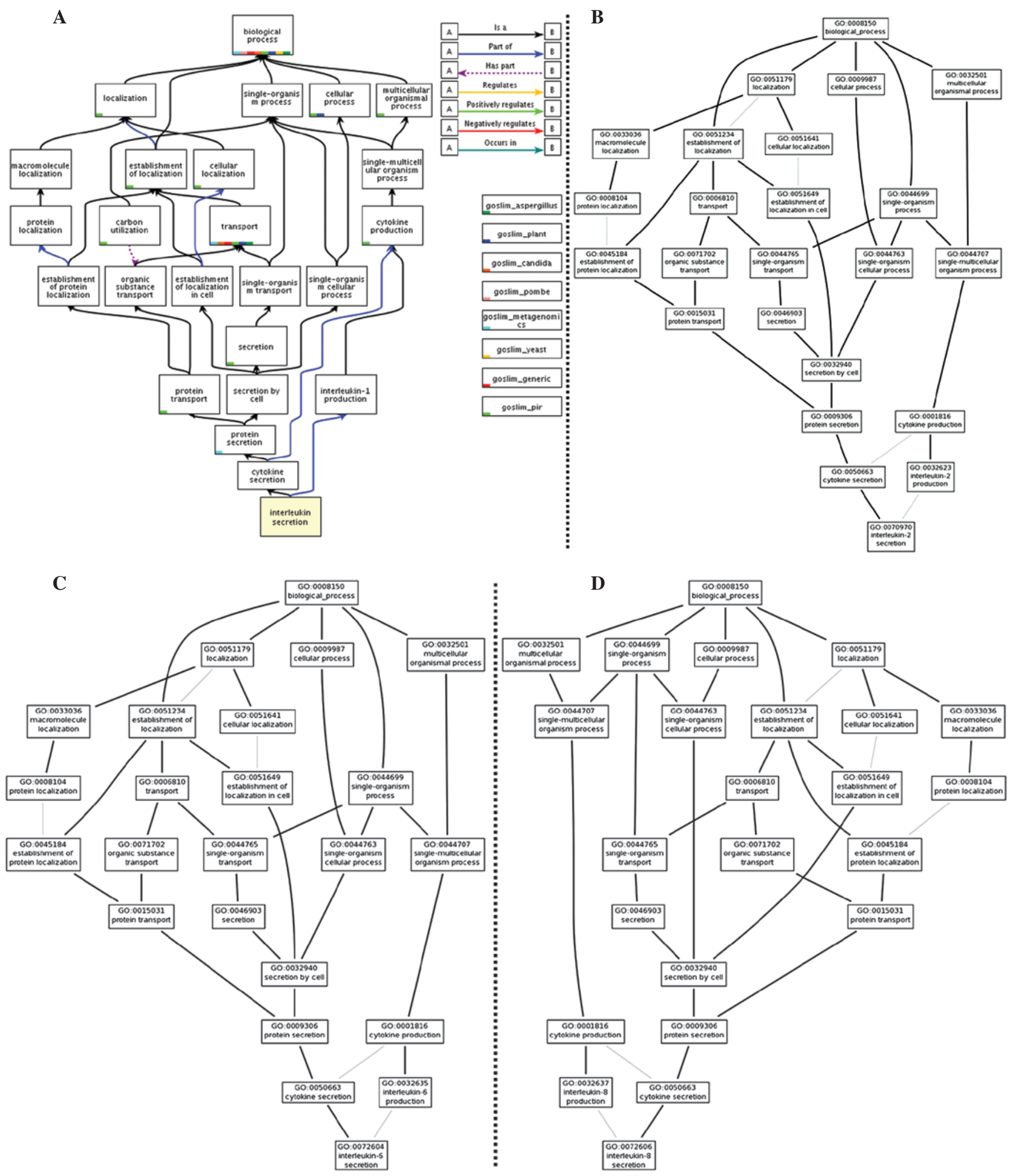

Figure 4. Canonical signaling pathways of inflammatory cytokines were generated using Gene Ontology (GO) software. (A) Generalization of inflammatory cytokine GO, (B) IL-2, (C) IL-6 and (D) IL-8. GO, gene ontology.

levels in invertebral disc herniation samples compared with samples from other disc disorders and control specimens. A previous study has demonstrated that inflammatory cytokines are produced by infiltrating mononuclear cells after the onset of disc herniation (14). Consistent with previous studies, increased levels of inflammatory cytokines were observed in the serum of patients with LDH compared with the control group.

Furthermore, the present study demonstrated that FLL ethanol extract may be used for the treatment of LDH. An et al (15) found that FLL treatment reduced inflammation via inhibition of nuclear factor- $\mathrm{\kappa} B$ in mouse peritoneal 

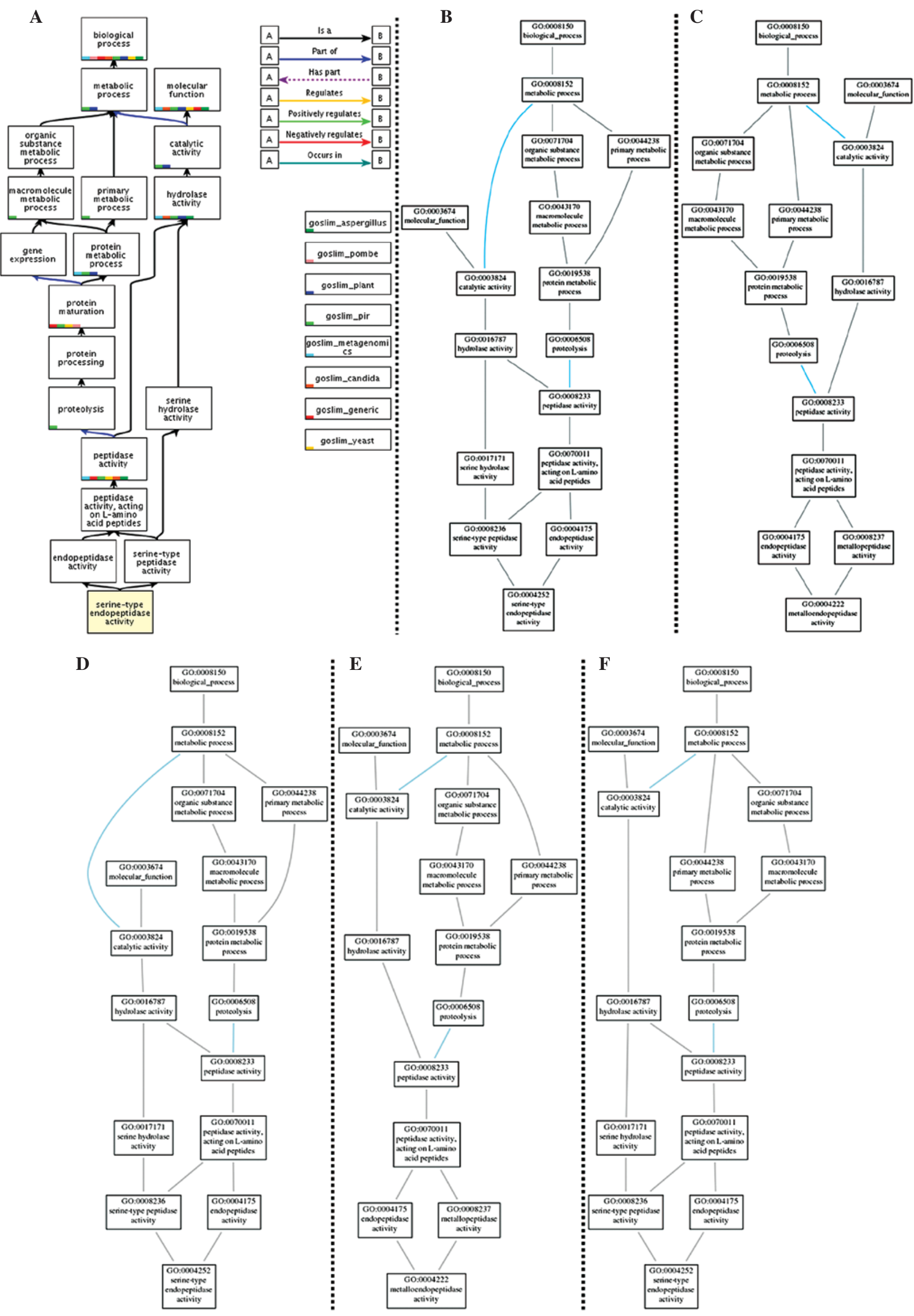

Figure 5. Canonical signaling pathways of MMPs were generated using GO software. (A) Generalization of MMPs, (B) MMP-1, (C) MMP-3, (D) MMP-8, (E) MMP-9 and (F) MMP-13. MMPs, metalloproteinase; GO, gene ontology; IL, interleukin. 
macrophages. The results of the present study also demonstrated that daily FLL ethanol extract treatment decreased the levels of inflammatory cytokines in serum in rats with LDH. Previous studies have also demonstrated that FLL promotes bone development with increased mineral density and improved bone mechanical properties $(16,17)$. It is hypothesized that bone development in response to FLL treatment may benefit LDH treatment. However, the mechanisms underlying these processes require further investigation.

Gene expression profiling and GO analysis were conducted in order to explore the changes of mRNA expression in the FLL-treated group. No changes among associated genes were observed. However, the results of the GO analyses suggested that FLL may be associated with a number of biological processes, including cytokine production and secretion, cellular components, including the proteasome, and molecular functions, including proteolysis. GO terms for cytokine secretion and cytokine production were highly enriched in the FLL treated group. Therefore, we focused further on cytokine secretion, which were induced by FLL. The limitation of this method is conventional gene expression signatures are simpler as it requires less transformation of the data. Therefore, the mechanistic meaning of their co-regulation remains difficult to interpret.

In conclusion, the present study was the first to demonstrate the potential of FLL treatment for LDH. The pharmacological effects and active ingredients of FLL should be investigated in future studies.

\section{Acknowledgements}

The authors would like to thank Dr Yu-Min Sun for technical assistance.

\section{References}

1. Li ML: Progress in the study on fructus Ligustri lucidi. Zhongguo Zhong Yao Za Zhi 19: 504-506, 1994 (In Chinese)

2. Zhang Y, Lai WP, Leung PC, Wu CF, Yao XS and Wong MS: Effects of Fructus Ligustri Lucidi extract on bone turnover and calcium balance in ovariectomized rats. Biol Pharm Bull 29: 291-296, 2006.
3. Zhang Y, Lai WP,Leung PC, Che CT and Wong MS: Improvement of $\mathrm{Ca}$ balance by Fructus Ligustri Lucidi extract in aged female rats. Osteoporos Int 19: 235-242, 2008.

4. Hoy D, March L, Brooks P, Woolf A, Blyth F, Vos T and Buchbinder R: Measuring the global burden of low back pain. Best Pract Res Clin Rheumatol 24: 155-165, 2010.

5. Golob AL and Wipf JE: Low back pain. Med Clin North Am 98: 405-428, 2014

6. Iwamoto J, Sato Y, Takeda T and Matsumoto H: The return to sports activity after conservative or surgical treatment in athletes with lumbar disc herniation. Am J Phys Med Rehabil 89: 1030-1035, 2010.

7. Daneyemez M, Sali A, Kahraman S, Beduk A and Seber N: Outcome analyses in 1072 surgically treated lumbar disc herniations. Minim Invasive Neurosurg 42: 63-68, 1999.

8. Findlay GF, Hall BI, Musa BS, Oliveira MD and Fear SC: A 10-year follow-up of the outcome of lumbar microdiscectomy. Spine (Phila Pa 1976) 23: 1168-1171, 1998.

9. Obata K, Tsujino H, Yamanaka H, Yi D, et al: Expression of neurotrophic factors in the dorsal root ganglion in a rat model of lumbar disc herniation. Pain 99: 121-132, 2002

10. Sasaki N, Sekiguchi M, Kikuchi S and Konno S: Anti-nociceptive effect of bovine milk-derived lactoferrin in a rat lumbar disc herniation model. Spine (Phila Pa 1976) 35: 1663-1667, 2010.

11. Zhang B, Schmoyer D, Kirov S and Snoddy J: GOTree Machine (GOTM): A web-based platform for interpreting sets of interesting genes using Gene Ontology hierarchies. BMC Bioinformatics 5: $16,2004$.

12. Weiler C, Nerlich AG, Zipperer J, Bachmeier BE and Boos N: 2002 SSE award competition in basic science: Expression of major matrix metalloproteinases is associated with intervertebral disc degradation and resorption. Eur Spine J 11: 308-320, 2002.

13. Tsarouhas A, Soufla G, Katonis P, Pasku D, Vakis A and Spandidos DA: Transcript levels of major MMPs and ADAMTS-4 in relation to the clinicopathological profile of patients with lumbar disc herniation. Eur Spine J 20: 781-790, 2011.

14. Yoshida M, Nakamura T, Sei A, Kikuchi T, Takagi K and Matsukawa A: Intervertebral disc cells produce tumor necrosis factor alpha, interleukin-1beta, and monocyte chemoattractant protein-1 immediately after herniation: An experimental study using a new hernia model. Spine (Phila Pa 1976) 30: 55-61, 2005.

15. An HJ, Jeong HJ, Um JY, Park YJ, Park RK, Kim EC, Na HJ, Shin TY, Kim HM and Hong SH: Fructus Ligustrum lucidi inhibits inflammatory mediator release through inhibition of nuclear factor-kappaB in mouse peritoneal macrophages. J Pharm Pharmacol 59: 1279-1285, 2007.

16. Feng X, Lyu Y, Wu Z, Fang Y, Xu H, Zhao P, Xu Y and Feng H: Fructus ligustri lucidi ethanol extract improves bone mineral density and properties through modulating calcium absorption-related gene expression in kidney and duodenum of growing rats. Calcif Tissue Int 94: 433-441, 2014.

17. Lyu Y, Feng X, Zhao P, et al: Fructus Ligustri Lucidi (FLL) ethanol extract increases bone mineral density and improves bone properties in growing female rats. J Bone Miner Metab 32: 616-626, 2014. 